\title{
Pulmonary edema because of incomplete hanging attempt
}

\author{
Cevdet Yardımcı ${ }^{1^{*}}$, Atilla Tekin ${ }^{2}$, Hilmi Demirkıran ${ }^{3}$ \\ ${ }^{1}$ Department of Anesthesiology and Reanimation, Cizre State Hospital, Sirnak, Turkey \\ ${ }^{2}$ Department of Pscyhiatry, Cizre State Hospital, Sirnak, Turkey \\ ${ }^{3}$ Department of Anesthesiology and Reanimation, V an Yuzuncu Yil University Medical Faculty, V an Turkey
}

\begin{abstract}
Hanging is used worlwide for suicide. It is generally ends with sudden death. But if the person is reached in a short time after hanging attempt, the patient can be saved. But we may come across some medical problems like pulmonary edema, cervical vertebra instability, impaired cognitive functions. 28 year old female patient had been taken to our hospital after incomplete hanging attempt with suicide intention. While she was in our hospital she had pulmonary complication. She had mechanical ventilation need. We supported her with mechanical ventilation both invasively and noninvasively. After 4 days of mechanical ventilation support she was discharged to the psychiatry clinic without any sequel.
\end{abstract}

Key Words: Incomplete hanging, suicide, pulmonary edema

\section{Introduction}

Hanging is one of the most frequent methods in the worldwide for suicide. If the individual doesn't die after hanging, some complications like cerebral hypoxic dysfunction, aspiration pneumonia and status epilepticus may ocur. We aimed to present pulmonary edema complication occuring in a patient after committing suicide by hanging herself.

\section{Case report}

A 28 year old female patient had been found in a room as hanged on a rope, and she had been saved from hanging rope by her family members. She has been taken to the state hospital and hospitalised by psychiatry department. After then she was consultated by our clinic. In her physical examination; general state was moderate, consciousness agitated-restless, tachypneic (RR: 44 respiration/minute), pulmonary crepitan ralles bilaterally and, hanging rope trace were observed in her neck (Figure 1), she was tachycardic (130 beat/minute), blood pressure: $140 / 90 \mathrm{mmHg}$, her lips were pale. SPO2 were $88 \%$ with oxygen 4 $\mathrm{L} /$ minute mask therapy. Arterial blood gas values were; $\mathrm{pH}=7.26, \quad \mathrm{pCO} 2=36 \mathrm{mmHg}, \quad \mathrm{OO} 2=62$ $\mathrm{mmHg}, \mathrm{HCO} 3=16 \mathrm{mEq} / \mathrm{L}, \mathrm{BE}=-10, \quad \mathrm{Lac}=3.2$
mmol/L. Noninvasive mechanical ventilation support was applied to the patient with bilateral widespread pulmonary edema on radiographic evaluation (Figure 2). In her follow up, desaturation was persisted in spite of noninvasive mechanical ventilation support. Then she was sedatized, curarized and intubated. She was mechanically ventilated invasively, in SIMV volume mod with f:12, Vt:500 ml, FiO2:\%60, PEEP:7 mmHg and I/E:1/2 ratio values. She was sedatized with continuos midazolam infusion. Pink frotty sputum was aspirated from intubation tube. Fluid restriction and mild diuresis with furosemide were applied to the patient. In her follow up the ralles were cleared, bilateral pulmonary sites were ventilating equally. On the following day sedation was stopped, the patient extubated herself during weaning. The patient who went tachypneic after self extubation was resedatized and reintubated when her ABG results got worse, then mechanically ventilated invasively. Pink frotty sputum was seen in the endotracheal tube, widespread crepitating ralles were heard bilaterally in the lung areas. Mild diuresis with furosemide were used. After 48 hours, ABG values were normal, the patient who hadn't crepitating ralles. Midazolam infusion ceased. Later, the patient was extubated. The patient was cooperated. But desaturated patient after extubation were supported with noninvasive 
mechanical ventilation in CPAP mode with FiO2:50\%, Psupport:8 cm H2O, PEEP:8 cm H2O values. Supportive therapy was continued about 24 hours. On the 4 th day the patient who didn't need mechanical ventilation support was followed with $2 \mathrm{~L} /$ minute nasal $\mathrm{O} 2$ therapy. Computerised brain tomographic evaluation were applied to the patient for cerebral injury control. The patient having no pathological findings was transferred to psychiatry clinic having no need of O2 support, conscious-cooperated, hemodynamically stable and normal $A B G$ values state.

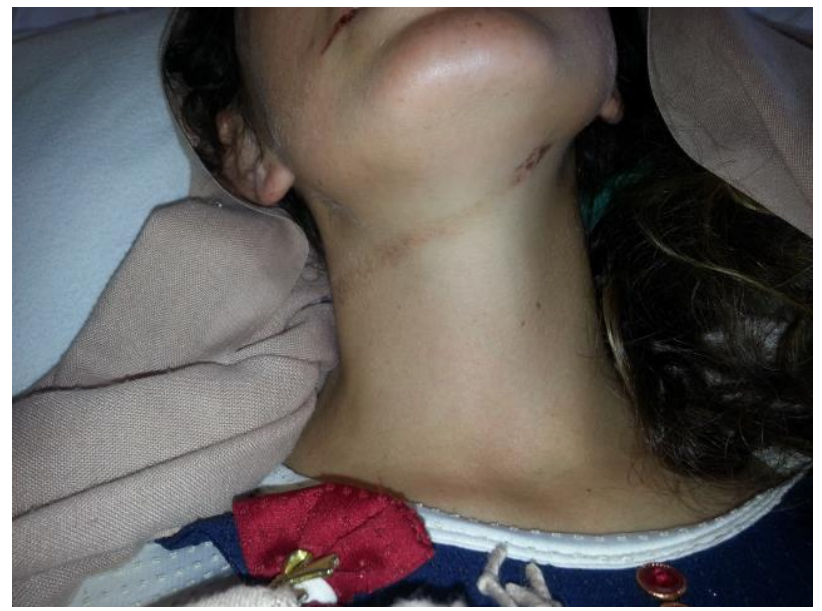

Fig. 1. Trace of hanging rope on the patient' s neck.

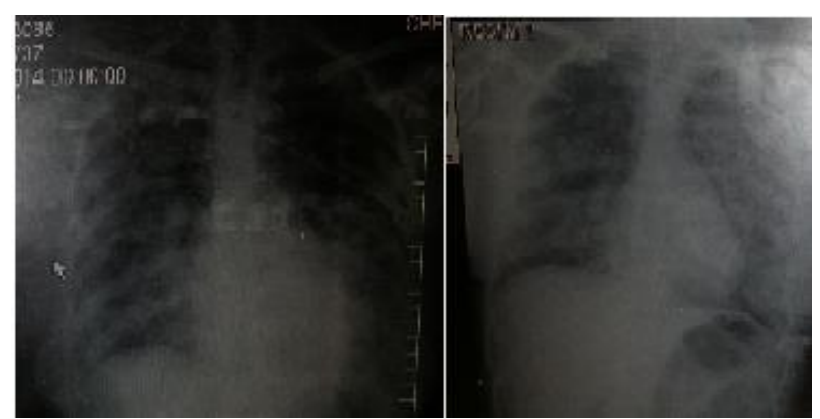

Fig. 2. Radiographic chest view of the patient in the figure 1.

\section{Discussion}

Strangulation is a well known topic in forensic science and there are lots of studies. It is guessed that $2.5 \%$ of all traumatic deaths in the world is because of strangulation. Hanging, is defined as pressure application to a person's neck partially or completely to a fully hanged. Hanging is one of the most frequent suicide method also in our country like in the world $(1,2)$.

Pathophysiology of morbidity and mortality due to hanging in literature;
- Venous blockade and cerebral hypoxia,

- Laryngeal edema and late phase airway blockade (due to loss of neck muscles tonus),

- Carotid sinus stimulation causing increased vagal tonus,

- Local damages (thyroid cartilage/hyoid bone fractures/laryngeal lacerations),

- Pulmonary complications (aspiration pneumonia, ARDS, pulmonary edema because of increased negative intrathoracic pressure or increased sympathic discharge from cental nervous system),

- Secondary cerebral injury (cerebral edema, diffuse cerebral hypoxemia and/or focal arterial dissection, arterial spasm or subarachoid hemorhage),

- Other complications (hypertermia, status epilepticus, carotid arter hemorage intramurally or esophageal tears) (1).

Death from hanging usually occurs beause of cerebral ischemia due to compression of airways and neck vasculature and sometimes cardiac arrest due to vagal inhibition occuring as a result of carotid compression. Sometimes the tounge may fall backward due to neck compression, blockades airways and mechanical asphyxia. But these mechanisms in hanging shouldn't be thought independently. All these mechanism act together and causes acute cerebral edema and death. Medulla spinalis injuries and cervical vertebra dislocations and fractures are usually seen in judicial hanging, in individuals who have serious degenerative cervical vertebral disease or in obese individuals falling abruptly $(2,3)$.

POPE (Post obstructive pulmonary edema) is a lifethreatening complication becoming after narrowing- relief of upper airway $(4,5)$. It is mainly seen in non-fatal hangings, but is reported as rare complication. Subpleural petechial hemorhages bilaterally, pink frotty edema fluid in trachea and hyperemia in other organs may happen after hanging $(2,6)$.

One of the theories for pulmonary edema occuring after narrowing and relieving of upper airways; is highly increased intrathoracic negative pressure during breathing effort against closed upper airways, causes return of excessive amount of blood to the thoracic cavity and heart. Hypoxia causes vasoconstriction and pulmonary vasculature permeability increase, both hypoxia and respiratory acidosis weaken heart contraction capacity. All of these conditions causes intrathoracic blood increase inducing pulmonary edema $(6,7)$. 
The other theory about pulmonary edema development after hanging is cathecolamine level rise after relief of upper airway. The main mechanism of neurogenic pulmonary edema is pulmonary severe vasoconstriction due to development of adrenegic response to cerebral injury and hypoxia, afterwards pulmonary capillary permeability increase develops $(8,9,10)$.

In a retrospective study made by Avni Kaya "Acute respiratory distress syndrom erelated to near hanging:a case report", it is reported that 3 of 23 incomplete hanging cases had acute respiratory distress syndrome (9).

In our case ARDS developed, and the patient healed without sequel applying mechanical ventilatory support therapy. In a case reported by K. Nishi et al. (10), they reported no neurologic cerebral injury; but they reported cathecolamine level rises probable "fight or flight" response. Also they mention that hypoxia can increase cathecolamine levels. Additionally they told that the mechanism happening in neurogenic pulmonary edema can contribute to pulmonary edema occurring after upper airway narrowing and relief. In our consious but agitated case we couldn't find any injury in computerised tomographic evaluation, and we couldn't differentiate the cause of agitation pulmonary edema whether because of hypoxia or sympathetic discharge due to cerebral damage in microscopic level. To differentiate the cause of pulmonary edema happening in these patients is very difficult, but it is very probable that both of the mechanisms contribute each other.

But what ever the mechanism of pulmonary edema in strangulation cases is, these patients can be treated without any sequel with sufficient oxygenation and mechanical ventilatory support.

Acknowlegment: This article was published as poster representation in the 21 th International care symposium, İstanbul, 2017.

\section{References}

1. Gandhi R, Taneja N, Mazumder P. Near hanging: Early intervention can save lives. Indian Journal of Anaesthesia 2011; 55: 388-391.

2. Yildırım M, Sivri S. Medico-legal examination of hanging. Journal of Clinical and Experimental Investigations 2015; 6: 400-405.

3. Cantürk C, Cantürk G. Deaths due to hanging in 1stanbul; evaluation of autopsies between 20002002 Journal of Forensic Medicine 2005; 19: 6-13.

4. Gluecker T, Capasso P, Schnyder P, et al. Clinical and radiologic features of pulmonary edema. Radiographics 1999; 19: 1507-1531.

5. Bhaskar B, Fraser JF. Negative pressure pulmonary edema revisited: Pathophysiology and review of management. Saudi J Anaesth 2011; 5: 308-313.

6. Demirci Ş, Doğan KH, Asphyxia types and dead body examination in asphyxia cases. Clinical Development journal 2009; 22: 23-32.

7. Balc1 $\mathrm{Y}$, Teyin $\mathrm{M}$, life threatening injury" evaluation in people who recovered from asphyxia J Foren Med 2004; 1: 84-92.

8. Adams N. Near hanging. Emerg Med 1999; 11: 17-21.

9. Kaya A, Okur M, Akbayram S. Acute respiratory distress syndromerelated to near hanging: a case report 2012; 47: 302-304.

10. Nishi K, Yanagawa Y, Hagiwara A. A case of pulmonary oedema induced by suffocation. West Indian Med J 2010; 59: 113-114. 